\title{
Allelopathic Effect of different Intercropping System and Tree Leaf Extract Spray on Weed Density, Dry Matter and Weed Control Efficiency in Irrigated Cotton
}

\author{
A. Sathishkumar ${ }^{1 *}$, G. Srinivasan ${ }^{2}$, T. $\operatorname{Ragavan}^{1}$, S. Thiyageshwari ${ }^{3}$ and N. Aananthi ${ }^{4}$ \\ ${ }^{1}$ Department of Agronomy, AC\&RI, Madurai-625104, Tamil Nadu, India \\ ${ }^{2}$ Department of Agronomy, ADAC\&RI, Trichy-09, Tamil Nadu, India \\ ${ }^{3}$ Department of Soils and Environment, Madurai-625104, Tamil Nadu, India \\ ${ }^{4}$ Department of Plant Breeding and Genetics, Madurai-625104, Tamil Nadu, India \\ *Corresponding author
}

\section{A B S T R A C T}

A field experiment was conducted at Agricultural College and Research Institute, Madurai to study the combined effect of intercropping system and weed management practices on

Keywords

Intercropping,

Weed management,

Weed density,

Weed control

efficiency.

Article Info

Accepted:

19 May 2017

Available Online:

10 June 2017 weed control in irrigated cotton during the year of 2016. Cotton + sorghum intercropping system registered significantly lower weed density and dry matter production at all the stages of crop growth, while sole cotton recorded the higher weed density and dry matter production. In weed management practices, significantly lesser weed density and dry matter were recorded under pre-emergence application of pendimethalin $1.0 \mathrm{~kg}$ a.i. $\mathrm{ha}^{-1}$ at 20 DAS. At 40 and 60 DAS hand weeding twice at 20 and 40 DAS recorded lower weed density and dry matter. Among the interaction effect, intercropping of cotton + sorghum and application of pendimethalin $1.0 \mathrm{~kg}$ a.i. ha $^{-1}$ significantly decreased the weed populations and dry matter at 20 DAS. At 40 and 60 DAS intercropping of cotton with sorghum and hand weeding twice at 20 and 40 DAS registered significantly lesser weed density and dry matter. The maximum weed density and dry matter was noticed under sole cotton with control. The higher weed control efficiency (WCE) of 81.2 per cent was obtained under the treatment of pre-emergence application of pendimethalin $1.0 \mathrm{~kg} \mathrm{a.i.} \mathrm{ha}{ }^{-1}$ at 20 DAS. At 40 and 60 DAS (86.3 and 94.4\%) WCE was higher with hand weeding twice at 20 and 40 DAS.

\section{Introduction}

Cotton the "white gold or the king of fibres" is one of the most important commercial crop in India. Cotton is known for the fibre and oil from seed, which plays a prominent role in the national and international economy. Initial slow growth and adoption of wider spacing favours the weeds to grow luxuriously in cotton fields. Manual weed management practice is laborious and expensive. In spite of herbicides being effective in increasing yield, indiscriminate use of herbicides has resulted in serious ecological implications such as development of herbicide resistance weeds and shifts in weed population. Recently, research attention has been focused on to find out alternative strategies for chemical weed control in several crops. Reduction in herbicide use is one of major goals of modern 
agriculture and there is much emphasis on search for alternative weed management strategies that are cheap, safe and sustainable. Allelopathy is considered as an effective, economical and environment friendly weed management approach (Iqbal and Cheema, 2009). Allelopathy is the releasing of Allelopathic compounds by one plant species that inhibit the growth and development of neighbouring plants of another species. Allelopathic potential in plants may be used in different ways to influence weed such as surface mulch, incorporation into the soil, spraying of leaf extracts, crop rotation, smothering or mixed cropping and intercropping. The slow initial growth coupled with indeterminate growth habit favours the growing of intercrops without affecting yield of cotton. It is indeed worthy to use land fully well by resorting to the introduction of intercrops. Intercropping has unique capacity to raise the unit profitability without disturbing the cotton ecosystem. Hence, the present investigation was carried out with the intercropping system and tree leaf extract on eco-friendly weed management in irrigated cotton.

\section{Materials and Methods}

A field experiment was conducted at Agricultural College and Research Institute, Madurai during 2016. Twenty four treatment combinations comprised of four intercropping, $\mathrm{I}_{1}$ - Cotton + Sorghum (1:1), $\mathrm{I}_{2}$ Cotton + Sunflower (1:1), $\mathrm{I}_{3}-$ Cotton + Sesame (1:1), $\mathrm{I}_{4}$ - Sole cotton and six weed management practices, $\mathrm{W}_{1}$ - PE Prosopis juliflora leaf extract @ 30\% + one hand weeding on 40 DAS, $\mathrm{W}_{2}$ - PE Annona squamosa leaf extract @ 30\% + one hand weeding on $40 \mathrm{DAS}, \mathrm{W}_{3}$ - PE Mangifera indica leaf extract @ 30\% + one hand weeding on $40 \mathrm{DAS}, \mathrm{W}_{4}$ - PE Pendimethalin @ $1.0 \mathrm{~kg}$ a.i. ha ${ }^{-1}+$ one hand weeding on 40 DAS, $\mathrm{W}_{5}-$ Two hand weeding at 20 and 40 DAS, $\mathrm{W}_{6}$ - Control (No weeding or spray).
The experiment was laid out in split plot design with three replications. Healthy and viable seeds of SVPR 4 cotton variety were sown as base crop at the rate of $15 \mathrm{~kg} \mathrm{ha}^{-1}$. Main cotton crop was sown with row to row spacing of $75 \mathrm{~cm}$ and plant to plant spacing of $30 \mathrm{~cm}$, on the same day intercrops were sown in between two rows of cotton crop following 1:1 ratio for main and intercrops. The plant to plant spacing adopted for intercrop was 30 cm. Leaves of Prosopis juliflora, Annona squamosa and Mangifera indica species at vegetative stage were collected and leaves were washed gently with tap water few seconds for removing contaminants like dust etc. The fresh leaves of above species cut into small species, soaked in alcohol and water@ 1:1 proportion and kept for overnight. After 12 hours, soaked leaves were ground with the help of mixer grinder. From the paste, the leaf extract of each botanical species was prepared by filtration which represented 100 per cent stock solution. From the stock solution, 30 per cent concentration was prepared and sprayed as per the treatment schedule. The weed control efficiency was worked out on the basis of weed populations.

$\operatorname{WCE}(\%)=\frac{\text { WDC-WDT }}{\text { WDC }}$

Where, $\mathrm{X}=$ Weed density in control plot $\left(\right.$ Number $\left.\mathrm{m}^{-2}\right), \mathrm{Y}=$ Weed density in treated plot (Number $\mathrm{m}^{-2}$ )

\section{Results and Discussion}

The weed flora observed in the experimental field was Dactyloctenium aegyptium, Echinochloa colonum and Chloris barbata among grasses, Cyperus rotundus among sedges and Trianthema portulacastrum, Amaranthus viridis, Cleome viscosa, Convolvulus arvensis, Corchorus trilocularis, Phyllanthus maderaspatensis and Digera arvensis among broad leaved weeds. The 
intercropping in cotton exerted significant influence on reducing the total weed populations and dry matter production (DMP) at 20, 40 and at 60 DAS. Among the intercropping system, cotton + sorghum reduced the weed density and DMP to a higher level and it was comparable with cotton + sesame.

The cotton + sorghum system registered lower weed density $(35.89,51.39$ and 36.83 $\left.\mathrm{m}^{-2}\right)$ and DMP $(149.53,261.33$ and $164.48 \mathrm{~kg}$ $\mathrm{ha}^{-1}$ ) at 20,40 and at 60 DAS, respectively. Sole cotton registered higher weed density (53.11, 75.39 and $57.15 \mathrm{~m}^{-2}$ ) and DMP $\left(189.62,334.05\right.$ and $\left.213.77 \mathrm{~kg} \mathrm{ha}^{-1}\right)$ at 20,40 and at 60 DAS, respectively. The intercropping suppressed the weed growth due to their spreading canopy coverage. The increased populations per unit area and crop competition in intercropping were also the possible reason for effective weed control.

The reduction of weed density might be due to Allelopathic compounds released by sorghum through root exudation. Significant reduction of weed density in cotton + sorghum intercropping system was reported by (Aladakatti et al., 2011).

The reduced dry weight under intercropping system could be attributed to high plant population and competitive ability of intercrops to effectively utilize resources from weeds and impeding weed growth by disturbing their physiological functions (Ion uptake, photosynthesis and respiration) through allelopathy (Oliveira et al., 2011).

Among the weed management practices, herbicides application recorded lower density of weeds at 20 DAS than control. Application of pendimethalin $1.0 \mathrm{~kg}$ a.i. ha ${ }^{-1}$ significantly reduced the weed populations and DMP to an intensity of $17.58 \mathrm{~m}^{-2}$ and $76.78 \mathrm{~kg} \mathrm{ha}^{-1}$, respectively. The reduced weed dry weight could be due to the reduction of weed density at early stages of crop growth. Application of pendimethalin, as pre-emergence spray was effective in weed control for cotton crop along with hand weeding (Tunio et al., 2003). The superiority of pendimethalin was also reported by (Singh and Kokate, 2010). At 40 and at $60 \mathrm{DAS}$, hand weeding twice at 20 and 40 DAS recorded the lower weed density (22.16 and $11.17 \mathrm{~m}^{-2}$ ) and DMP (145.10 and $43.40 \mathrm{~kg} \mathrm{ha}^{-1}$ ). It might have been due to removal of weed plants by manual weeding and chances of establishment of new weeds were reduced because of smothering effect of crop over weeds.

The higher weed density and DMP was recorded under control. Among the leaf extract spray, pre-emergence (PE) application of Mangifera indica leaf extract at 30 per cent recorded lesser weed populations (24.84, 44.25 and $23.50 \mathrm{~m}^{-2}$ ) and DMP (121.25, 230.58 and $108.28 \mathrm{~kg} \mathrm{ha}^{-1}$ ) at 20, 40 and at 60 DAS, respectively compared to control. The extract of mango leaves has the capacity of killing or suppressing weed growth (Rudramuni et al., 2006). Mango leaves have been reported to contain many different kinds of phenolic compounds like, Ferulic, coumaric, benzoic, vanelic, chlorogenic, caffiec, Gallic, hydroxybenzoic and cinnamic (El-Rokiek et al., 2010).

The interaction effect was significant between intercropping and weed management practices at 20, 40 and at 60 DAS. The combination of cotton + sorghum intercropping system and application of pendimethalin $1.0 \mathrm{~kg} \mathrm{ha}^{-1}$ a.i. $\mathrm{ha}^{-1}$ was more efficient in reducing the weed density and DMP $\left(13.00 \mathrm{~m}^{-2}\right.$ and $\left.67.10 \mathrm{~kg} \mathrm{ha}^{-1}\right)$ at 20 DAS. At 40 and 60 DAS, intercropping of cotton + sorghum along with hand weeding twice at 20 and 40 DAS registered significantly lower weed density (17.66 and $9.33 \mathrm{~m}^{-2}$ ) and DMP (133.40 and $\left.39.30 \mathrm{~kg} \mathrm{ha}^{-1}\right)$. 
Table.1 Effect of intercropping system and weed management practices on total weed density $\left(\mathrm{m}^{-2}\right)$ in cotton

\begin{tabular}{|c|c|c|c|c|c|c|c|c|c|c|c|c|c|c|c|}
\hline & \multicolumn{5}{|c|}{$20 \mathrm{DAS}$} & \multicolumn{5}{|c|}{40 DAS } & \multicolumn{5}{|c|}{$60 \mathrm{DAS}$} \\
\hline Treatment & $\mathrm{I}_{\mathrm{I}}$ & $\mathrm{I}_{2}$ & $\mathrm{I}_{3}$ & $\mathrm{I}_{4}$ & Mean & $\mathrm{I}_{\mathrm{I}}$ & $\mathrm{I}_{2}$ & $\mathrm{I}_{3}$ & $\mathrm{I}_{4}$ & Mean & $\mathrm{I}_{\mathrm{I}}$ & $\mathrm{I}_{2}$ & $\mathrm{I}_{3}$ & $\mathrm{I}_{4}$ & Mean \\
\hline $\mathrm{W}_{1}$ & $\begin{array}{c}5.67 \\
(31.67)\end{array}$ & $\begin{array}{c}5.82 \\
(33.33)\end{array}$ & $\begin{array}{c}5.76 \\
(32.67)\end{array}$ & $\begin{array}{c}6.39 \\
(40.33)\end{array}$ & $\begin{array}{c}5.91 \\
(34.50)\end{array}$ & $\begin{array}{c}7.24 \\
(51.99)\end{array}$ & $\begin{array}{c}8.24 \\
(67.33)\end{array}$ & $\begin{array}{c}7.56 \\
(56.66)\end{array}$ & $\begin{array}{c}9.23 \\
(84.67)\end{array}$ & $\begin{array}{c}8.07 \\
(65.16)\end{array}$ & $\begin{array}{c}5.12 \\
(25.67)\end{array}$ & $\begin{array}{c}5.52 \\
(30.00)\end{array}$ & $\begin{array}{c}5.46 \\
(29.33)\end{array}$ & $\begin{array}{c}6.34 \\
(39.66)\end{array}$ & $\begin{array}{c}5.61 \\
(31.17)\end{array}$ \\
\hline $\mathrm{W}_{2}$ & $\begin{array}{c}5.93 \\
(34.67)\end{array}$ & $\begin{array}{c}6.10 \\
(36.66)\end{array}$ & $\begin{array}{c}6.07 \\
(36.33)\end{array}$ & $\begin{array}{c}6.77 \\
(45.34)\end{array}$ & $\begin{array}{c}6.22 \\
(38.25)\end{array}$ & $\begin{array}{c}8.32 \\
(68.67)\end{array}$ & $\begin{array}{c}8.65 \\
(74.33)\end{array}$ & $\begin{array}{c}8.46 \\
(71.00)\end{array}$ & $\begin{array}{c}9.77 \\
(95.01)\end{array}$ & $\begin{array}{c}8.80 \\
(77.25)\end{array}$ & $\begin{array}{c}5.76 \\
(32.67)\end{array}$ & $\begin{array}{c}5.90 \\
(34.34)\end{array}$ & $\begin{array}{c}5.84 \\
(33.66)\end{array}$ & $\begin{array}{c}6.77 \\
(45.33)\end{array}$ & $\begin{array}{c}6.07 \\
(36.50)\end{array}$ \\
\hline $\mathrm{W}_{3}$ & $\begin{array}{c}4.74 \\
(21.99)\end{array}$ & $\begin{array}{c}5.08 \\
(25.34)\end{array}$ & $\begin{array}{c}4.92 \\
(23.67)\end{array}$ & $\begin{array}{c}5.37 \\
(28.35)\end{array}$ & $\begin{array}{c}5.03 \\
(24.84)\end{array}$ & $\begin{array}{c}6.23 \\
(38.33)\end{array}$ & $\begin{array}{c}6.89 \\
(47.01)\end{array}$ & $\begin{array}{c}6.54 \\
(42.33)\end{array}$ & $\begin{array}{c}7.06 \\
(49.34)\end{array}$ & $\begin{array}{c}6.68 \\
(44.25)\end{array}$ & $\begin{array}{c}4.74 \\
(21.99)\end{array}$ & $\begin{array}{c}4.88 \\
(23.33)\end{array}$ & $\begin{array}{c}4.78 \\
(22.33)\end{array}$ & $\begin{array}{c}5.18 \\
(26.34)\end{array}$ & $\begin{array}{c}4.90 \\
(23.50)\end{array}$ \\
\hline $\mathrm{W}_{4}$ & $\begin{array}{c}3.67 \\
(13.00)\end{array}$ & $\begin{array}{c}4.53 \\
(20.00)\end{array}$ & $\begin{array}{c}4.06 \\
(15.99)\end{array}$ & $\begin{array}{c}4.67 \\
(21.32)\end{array}$ & $\begin{array}{c}4.23 \\
(17.58)\end{array}$ & $\begin{array}{c}5.31 \\
(27.66)\end{array}$ & $\begin{array}{c}5.85 \\
(33.67)\end{array}$ & $\begin{array}{c}5.64 \\
(31.34)\end{array}$ & $\begin{array}{c}6.07 \\
(36.33)\end{array}$ & $\begin{array}{c}5.72 \\
(32.25)\end{array}$ & $\begin{array}{c}4.02 \\
(15.66)\end{array}$ & $\begin{array}{c}4.26 \\
(17.66)\end{array}$ & $\begin{array}{c}4.26 \\
(17.66)\end{array}$ & $\begin{array}{c}4.41 \\
(18.99)\end{array}$ & $\begin{array}{c}4.24 \\
(17.49)\end{array}$ \\
\hline $\mathrm{W}_{5}$ & $\begin{array}{c}7.34 \\
(53.33)\end{array}$ & $\begin{array}{c}7.63 \\
(57.66)\end{array}$ & $\begin{array}{c}7.38 \\
(54.00)\end{array}$ & $\begin{array}{c}9.50 \\
(89.66)\end{array}$ & $\begin{array}{c}7.96 \\
(63.66)\end{array}$ & $\begin{array}{c}4.26 \\
(17.66)\end{array}$ & $\begin{array}{c}4.85 \\
(23.00)\end{array}$ & $\begin{array}{c}4.78 \\
(22.33)\end{array}$ & $\begin{array}{c}5.11 \\
(25.66)\end{array}$ & $\begin{array}{c}4.75 \\
(22.16)\end{array}$ & $\begin{array}{c}3.14 \\
(9.33)\end{array}$ & $\begin{array}{c}3.39 \\
(11.00)\end{array}$ & $\begin{array}{c}3.39 \\
(11.00)\end{array}$ & $\begin{array}{c}3.72 \\
(13.33)\end{array}$ & $\begin{array}{c}3.41 \\
(11.17)\end{array}$ \\
\hline $\mathrm{W}_{6}$ & $\begin{array}{c}7.82 \\
(60.67)\end{array}$ & $\begin{array}{c}8.28 \\
(68.01)\end{array}$ & $\begin{array}{c}8.05 \\
(64.34)\end{array}$ & $\begin{array}{c}9.70 \\
(93.66)\end{array}$ & $\begin{array}{c}8.46 \\
(71.67)\end{array}$ & $\begin{array}{c}10.22 \\
(104.00)\end{array}$ & $\begin{array}{c}11.34 \\
(128.00)\end{array}$ & $\begin{array}{c}10.95 \\
(119.33)\end{array}$ & $\begin{array}{c}12.72 \\
(161.34)\end{array}$ & $\begin{array}{c}11.31 \\
(128.17)\end{array}$ & $\begin{array}{c}10.78 \\
(115.68)\end{array}$ & $\begin{array}{c}11.68 \\
(136.00)\end{array}$ & $\begin{array}{c}11.37 \\
(128.67)\end{array}$ & $\begin{array}{c}14.13 \\
(199.27)\end{array}$ & $\begin{array}{c}11.99 \\
(144.91)\end{array}$ \\
\hline \multirow[t]{2}{*}{ Mean } & $\begin{array}{c}5.86 \\
(35.89) \\
\end{array}$ & $\begin{array}{c}6.24 \\
(40.17) \\
\end{array}$ & $\begin{array}{c}6.04 \\
(37.83) \\
\end{array}$ & $\begin{array}{c}7.07 \\
(53.11) \\
\end{array}$ & & $\begin{array}{c}6.93 \\
(51.39) \\
\end{array}$ & $\begin{array}{c}7.64 \\
(62.22) \\
\end{array}$ & $\begin{array}{c}7.32 \\
(57.17) \\
\end{array}$ & $\begin{array}{c}8.33 \\
(75.39) \\
\end{array}$ & & $\begin{array}{c}10.78 \\
(36.83) \\
\end{array}$ & $\begin{array}{c}11.68 \\
(42.06) \\
\end{array}$ & $\begin{array}{c}11.37 \\
(40.44) \\
\end{array}$ & $\begin{array}{c}14.13 \\
(57.15) \\
\end{array}$ & \\
\hline & I & $\mathrm{W}$ & I at W & $\mathrm{W}$ at I & & I & $\mathrm{W}$ & I at W & $\mathrm{W}$ at I & & I & $\mathrm{W}$ & I at W & $\mathrm{W}$ at I & \\
\hline $\mathrm{CD}(\mathrm{p}=0.05)$ & 0.26 & 0.26 & 0.54 & 0.52 & & 0.40 & 0.34 & 0.74 & 0.69 & & 0.35 & 0.51 & 1.00 & 1.03 & \\
\hline
\end{tabular}

Figures in the parenthesis are original values. Others are $\sqrt{ }(\mathrm{X}+0.5)$.

$\mathrm{I}_{1}$ - Cotton + Sorghum (1:1), $\mathrm{I}_{2}$ - Cotton + Sunflower (1:1), I $\mathrm{I}_{3}$ - Cotton + Sesame (1:1), $\mathrm{I}_{4}$ - Sole cotton, W - PE Prosopis juliflora leaf extract @ 30\% + one HW on 40 DAS, $\mathrm{W}_{2}$ - PE Annona squamosa leaf extract @ 30\% + one HW on 40 DAS, $\mathrm{W}_{3}-\mathrm{PE}$ Mangifera indica leaf extract @ $30 \%+$ one HW on 40 DAS, W 4 -

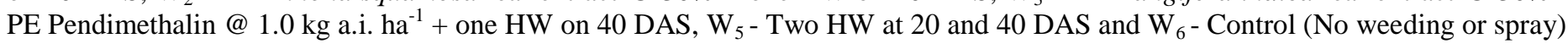


Int.J.Curr.Microbiol.App.Sci (2017) 6(6): 1322-1329

Table.2 Effect of intercropping system and weed management practices on total weed dry matter production (kg ha-1) in cotton

\begin{tabular}{|c|c|c|c|c|c|c|c|c|c|c|c|c|c|c|c|}
\hline \multirow[b]{2}{*}{ Treatment } & \multicolumn{5}{|c|}{$20 \mathrm{DAS}$} & \multicolumn{5}{|c|}{$40 \mathrm{DAS}$} & \multicolumn{5}{|c|}{$60 \mathrm{DAS}$} \\
\hline & $\mathrm{I}_{\mathrm{I}}$ & $\mathrm{I}_{2}$ & $\mathrm{I}_{3}$ & $\mathrm{I}_{4}$ & Mean & $\mathrm{I}_{\mathrm{I}}$ & $\mathrm{I}_{2}$ & $\mathrm{I}_{3}$ & $\mathrm{I}_{4}$ & Mean & $\mathrm{I}_{\mathrm{I}}$ & $\mathrm{I}_{2}$ & $\mathrm{I}_{3}$ & $\mathrm{I}_{4}$ & Mean \\
\hline $\mathrm{W}_{1}$ & $\begin{array}{c}11.89 \\
(140.80)\end{array}$ & $\begin{array}{c}12.32 \\
(151.20)\end{array}$ & $\begin{array}{c}12.01 \\
(143.80)\end{array}$ & $\begin{array}{c}13.88 \\
(192.10)\end{array}$ & $\begin{array}{c}12.53 \\
(156.98)\end{array}$ & $\begin{array}{c}16.30 \\
(265.20)\end{array}$ & $\begin{array}{c}17.26 \\
(297.40)\end{array}$ & $\begin{array}{c}16.71 \\
(278.80)\end{array}$ & $\begin{array}{c}20.38 \\
(414.80)\end{array}$ & $\begin{array}{c}17.66 \\
(314.05)\end{array}$ & $\begin{array}{c}11.32 \\
(127.6 \\
0)\end{array}$ & $\begin{array}{c}11.75 \\
(137.60)\end{array}$ & $\begin{array}{c}11.47 \\
(131.00)\end{array}$ & $\begin{array}{c}13.48 \\
(181.30)\end{array}$ & $\begin{array}{c}12.01 \\
(144.38)\end{array}$ \\
\hline $\mathrm{W}_{2}$ & $\begin{array}{c}12.82 \\
(163.90)\end{array}$ & $\begin{array}{c}13.36 \\
(178.00)\end{array}$ & $\begin{array}{c}13.07 \\
(170.40)\end{array}$ & $\begin{array}{c}14.43 \\
(207.80)\end{array}$ & $\begin{array}{c}13.42 \\
(180.03)\end{array}$ & $\begin{array}{c}17.59 \\
(308.90)\end{array}$ & $\begin{array}{c}19.76 \\
(390.10)\end{array}$ & $\begin{array}{c}18.79 \\
(352.40)\end{array}$ & $\begin{array}{c}21.05 \\
(442.40)\end{array}$ & $\begin{array}{c}19.30 \\
(373.45)\end{array}$ & $\begin{array}{c}11.78 \\
(138.3 \\
0)\end{array}$ & $\begin{array}{c}12.86 \\
(164.90)\end{array}$ & $\begin{array}{c}12.19 \\
(148.10)\end{array}$ & $\begin{array}{c}13.84 \\
(191.10)\end{array}$ & $\begin{array}{c}12.67 \\
(160.60)\end{array}$ \\
\hline $\mathrm{W}_{3}$ & $\begin{array}{c}10.29 \\
(105.30)\end{array}$ & $\begin{array}{c}11.44 \\
(130.40)\end{array}$ & $\begin{array}{c}10.74 \\
(114.80)\end{array}$ & $\begin{array}{c}11.62 \\
(134.50)\end{array}$ & $\begin{array}{c}11.02 \\
(121.25)\end{array}$ & $\begin{array}{c}14.79 \\
(218.30)\end{array}$ & $\begin{array}{c}15.17 \\
(229.60)\end{array}$ & $\begin{array}{c}14.90 \\
(221.50)\end{array}$ & $\begin{array}{c}15.92 \\
(252.90)\end{array}$ & $\begin{array}{c}15.20 \\
(230.58)\end{array}$ & $\begin{array}{c}9.65 \\
(92.60)\end{array}$ & $\begin{array}{c}10.69 \\
(113.70)\end{array}$ & $\begin{array}{c}10.43 \\
(108.20)\end{array}$ & $\begin{array}{c}10.91 \\
(118.60)\end{array}$ & $\begin{array}{c}10.42 \\
(108.28)\end{array}$ \\
\hline $\mathrm{W}_{4}$ & $\begin{array}{c}8.22 \\
(67.10)\end{array}$ & $\begin{array}{c}8.74 \\
(75.90)\end{array}$ & $\begin{array}{c}8.41 \\
(70.30)\end{array}$ & $\begin{array}{c}9.71 \\
(93.80)\end{array}$ & $\begin{array}{c}8.77 \\
(76.78)\end{array}$ & $\begin{array}{c}13.78 \\
(189.40)\end{array}$ & $\begin{array}{c}13.93 \\
(193.50)\end{array}$ & $\begin{array}{c}13.85 \\
(191.30)\end{array}$ & $\begin{array}{c}14.00 \\
(195.60)\end{array}$ & $\begin{array}{c}13.89 \\
(192.45)\end{array}$ & $\begin{array}{c}7.31 \\
(53.00)\end{array}$ & $\begin{array}{c}7.97 \\
(63.00)\end{array}$ & $\begin{array}{c}7.71 \\
(58.90)\end{array}$ & $\begin{array}{c}8.87 \\
(78.10)\end{array}$ & $\begin{array}{c}7.97 \\
(63.25)\end{array}$ \\
\hline $\mathrm{W}_{5}$ & $\begin{array}{c}14.23 \\
(201.90)\end{array}$ & $\begin{array}{c}14.61 \\
(213.00)\end{array}$ & $\begin{array}{c}14.39 \\
(206.60)\end{array}$ & $\begin{array}{c}15.82 \\
(249.90)\end{array}$ & $\begin{array}{c}14.76 \\
(217.85)\end{array}$ & $\begin{array}{c}11.57 \\
(133.40)\end{array}$ & $\begin{array}{c}12.21 \\
(148.60)\end{array}$ & $\begin{array}{c}11.95 \\
(142.20)\end{array}$ & $\begin{array}{c}12.52 \\
(156.20)\end{array}$ & $\begin{array}{c}12.06 \\
(145.10)\end{array}$ & $\begin{array}{c}6.31 \\
(39.30)\end{array}$ & $\begin{array}{c}6.72 \\
(44.70)\end{array}$ & $\begin{array}{c}6.53 \\
(42.20)\end{array}$ & $\begin{array}{c}6.92 \\
(47.40)\end{array}$ & $\begin{array}{c}6.62 \\
(43.40)\end{array}$ \\
\hline $\mathrm{W}_{6}$ & $\begin{array}{c}14.79 \\
(218.20)\end{array}$ & $\begin{array}{c}15.16 \\
(229.20)\end{array}$ & $\begin{array}{c}14.95 \\
(223.10)\end{array}$ & $\begin{array}{c}16.13 \\
(259.60)\end{array}$ & $\begin{array}{c}15.26 \\
(232.53)\end{array}$ & $\begin{array}{c}21.29 \\
(452.80)\end{array}$ & $\begin{array}{c}22.37 \\
(499.90)\end{array}$ & $\begin{array}{c}21.70 \\
(470.20)\end{array}$ & $\begin{array}{c}23.30 \\
(542.40)\end{array}$ & $\begin{array}{c}22.17 \\
(491.33)\end{array}$ & $\begin{array}{c}23.16 \\
(536.1 \\
0)\end{array}$ & $\begin{array}{c}24.41 \\
(595.30)\end{array}$ & $\begin{array}{c}23.66 \\
(559.10)\end{array}$ & $\begin{array}{c}25.82 \\
(666.10)\end{array}$ & $\begin{array}{c}24.26 \\
(589.15)\end{array}$ \\
\hline Mean & $\begin{array}{c}12.04 \\
(149.53)\end{array}$ & $\begin{array}{c}12.61 \\
(162.95)\end{array}$ & $\begin{array}{c}12.26 \\
(154.83)\end{array}$ & $\begin{array}{c}13.60 \\
(189.62)\end{array}$ & & $\begin{array}{c}15.89 \\
(261.33)\end{array}$ & $\begin{array}{c}16.78 \\
(293.18)\end{array}$ & $\begin{array}{c}16.32 \\
(276.07)\end{array}$ & $\begin{array}{c}17.86 \\
(334.05)\end{array}$ & & $\begin{array}{c}11.59 \\
(164.4 \\
8)\end{array}$ & $\begin{array}{c}12.40 \\
(186.53)\end{array}$ & $\begin{array}{c}12.00 \\
(174.58)\end{array}$ & $\begin{array}{c}13.31 \\
(213.77)\end{array}$ & \\
\hline & I & $\mathrm{W}$ & I at W & $\mathrm{W}$ at I & & I & W & I at W & $\mathrm{W}$ at I & & I & W & I at W & $\mathrm{W}$ at I & \\
\hline $\begin{array}{c}C D \\
(p=0.05)\end{array}$ & 0.28 & 0.21 & 0.48 & 0.42 & & 0.70 & 0.69 & 1.44 & 1.38 & & 0.47 & 0.37 & 0.82 & 0.74 & \\
\hline
\end{tabular}

Figures in the parenthesis are original values. Others are $\sqrt{ }(X+0.5)$.

$\mathrm{I}_{1}$ - Cotton + Sorghum (1:1), $\mathrm{I}_{2}$ - Cotton + Sunflower (1:1), $\mathrm{I}_{3}$ - Cotton + Sesame (1:1), $\mathrm{I}_{4^{-}}$- Sole cotton, $\mathrm{W}_{1}$ - PE Prosopis juliflora leaf extract @ 30\% + one HW on 40 DAS, $\mathrm{W}_{2}$ - PE Annona squamosa leaf extract @ 30\% + one HW on 40 DAS, W ${ }_{3}$ - PE Mangifera indica leaf extract @ 30\% + one HW on 40 DAS, W 4 PE Pendimethalin @ $1.0 \mathrm{~kg}$ a.i. ha ${ }^{-1}+$ one HW on 40 DAS, $\mathrm{W}_{5}$ - Two HW at 20 and 40 DAS and $\mathrm{W}_{6}$ - Control (No weeding or spray) 


\section{Int.J.Curr.Microbiol.App.Sci (2017) 6(6): 1322-1329}

Table.3 Effect of intercropping system and weed management practices on weed control efficiency (\%) of cotton

\begin{tabular}{|c|c|c|c|c|c|c|c|c|c|c|c|c|c|c|c|}
\hline \multirow[b]{2}{*}{ Treatment } & \multicolumn{5}{|c|}{$20 \mathrm{DAS}$} & \multicolumn{5}{|c|}{$40 \mathrm{DAS}$} & \multicolumn{5}{|c|}{$60 \mathrm{DAS}$} \\
\hline & $\mathrm{I}_{\mathrm{I}}$ & $\mathrm{I}_{2}$ & $\mathrm{I}_{3}$ & $\mathrm{I}_{4}$ & Mean & $\mathrm{I}_{\mathrm{I}}$ & $\mathrm{I}_{2}$ & $\mathrm{I}_{3}$ & $\mathrm{I}_{4}$ & Mean & $\mathrm{I}_{\mathrm{I}}$ & $\mathrm{I}_{2}$ & $\mathrm{I}_{3}$ & $\mathrm{I}_{4}$ & Mean \\
\hline $\mathrm{W}_{1}$ & 66.2 & 64.4 & 65.1 & 56.9 & 63.2 & 67.8 & 58.3 & 64.9 & 47.5 & 59.6 & 87.1 & 84.9 & 85.3 & 80.1 & 84.4 \\
\hline $\mathrm{W}_{2}$ & 63.0 & 60.9 & 61.2 & 51.6 & 59.2 & 57.4 & 53.9 & 56.0 & 41.1 & 52.1 & 83.6 & 82.8 & 83.1 & 77.3 & 81.7 \\
\hline $\mathrm{W}_{3}$ & 76.5 & 72.9 & 74.7 & 69.7 & 73.5 & 76.2 & 70.9 & 73.8 & 69.4 & 72.6 & 89.0 & 88.3 & 88.8 & 86.8 & 88.2 \\
\hline $\mathrm{W}_{4}$ & 86.1 & 78.6 & 82.9 & 77.2 & 81.2 & 82.9 & 79.1 & 80.6 & 77.5 & 80.0 & 92.1 & 91.1 & 91.1 & 90.5 & 91.2 \\
\hline $\mathrm{W}_{5}$ & 43.1 & 38.4 & 42.3 & 4.3 & 32.0 & 89.1 & 85.7 & 86.2 & 84.1 & 86.3 & 95.3 & 94.5 & 94.5 & 93.3 & 94.4 \\
\hline $\mathrm{W}_{6}$ & 35.2 & 27.4 & 31.3 & - & 23.5 & 35.5 & 20.7 & 26.0 & - & 20.6 & 41.9 & 31.8 & 35.4 & - & 27.3 \\
\hline Mean & 61.7 & 57.1 & 59.6 & 43.3 & & 68.2 & 61.4 & 64.6 & 53.3 & & 81.5 & 78.9 & 79.7 & 71.3 & \\
\hline
\end{tabular}

*Data not statistically analysed

$\mathrm{I}_{1}$ - Cotton + Sorghum (1:1), $\mathrm{I}_{2}$ - Cotton + Sunflower (1:1), $\mathrm{I}_{3}$ - Cotton + Sesame (1:1), $\mathrm{I}_{4}$ - Sole cotton, W ${ }_{1}-\mathrm{PE} \mathrm{Prosopis} \mathrm{juliflora} \mathrm{leaf} \mathrm{extract} \mathrm{@} \mathrm{30 \%} \mathrm{+} \mathrm{one} \mathrm{HW}$ on 40 DAS, W - PE Annona squamosa leaf extract @ 30\% + one HW on 40 DAS, W - PE Mangifera indica leaf extract @ 30\% + one HW on 40 DAS, W 4 PE Pendimethalin@ $1.0 \mathrm{~kg}$ a.i. ha ${ }^{-1}+$ one HW on 40 DAS, $\mathrm{W}_{5}-$ Two HW at 20 and 40 DAS and $\mathrm{W}_{6}$ - Control (No weeding or spray) 
The maximum weed density and DMP was registered under sole cotton with control. Different intercropping system and tree leaf extract spray exhibited variation in weeds populations and DMP. Among the interaction effect, intercropping of cotton + sorghum and pre-emergence application of Mangifera indica leaf extract at $30 \%+$ hand weeding at 40 DAS was found to be most effective in reducing the total weed density $(21.99,38.33$, $21.99 \mathrm{~m}^{-2}$ ) and DMP 105.30, 218.30, 92.60 $\mathrm{kg} \mathrm{ha}^{-1}$ ) at 20, 40 and at 60 DAS, respectively. Among the interaction effect cotton + sorghum (1:1) and pre-emergence application of Mangifera indica leaf extract at $30 \%+$ hand weeding at 40 DAS $\left(\mathrm{I}_{1} \mathrm{~W}_{3}\right)$ was found to be most effective in reducing the total weed density $\left(21.99,38.33,21.99 \mathrm{~m}^{-2}\right.$ ) and DMP 105.30, 218.30, $\left.92.60 \mathrm{~kg} \mathrm{ha}^{-1}\right)$ at 20, 40 and 60 DAS, respectively) (Tables 1 and 2). This was followed by cotton + sesame $(1: 1)$ and pre-emergence application of Mangifera at indica leaf extracts $30 \%$ along with hand weeding at 40 DAS.

Cotton + sorghum intercropping system recorded the higher weed control efficiency (WCE) of 67.1, 68.2 and 81.5 per cent at 20, 40 and at 60 DAS. This was followed by cotton + sesame intercropping system. The sole cotton registered comparatively lower WCE. This remarkable reduction of weeds under intercropping systems could be correlated with reduced germination of weeds, low weed density and low biomass of weeds due to effective utilization of resources by crops and reduction of germination and growth of weeds through releasing Allelopathic compounds by intercrops (Iqba et al., 2007). Among the weed management practices, higher WCE of $86.1 \%$ was recorded in pre-emergence application of pendimethalin $1.0 \mathrm{~kg}$ a.i. $\mathrm{ha}^{-1}$ at $20 \mathrm{DAS}$. The hand weeding twice at 20 and 40 DAS recorded higher WCE (86.3 and $94.4 \%$ ) at 40 and 60 DAS. This might be due to lesser weed competition by the hand weeding which favoured the growth and development of cotton, thereby higher weed control efficiency was obtained at 40 and 60 DAS than other practices (Nithya and Chinnusamy, 2013). With respect to leaf extract spray, application of Mangifera at indica leaf extract $30 \%+$ hand weeding at 40 DAS recorded higher WCE $(73.5,72.6$ and $88.2 \%$ at 20,40 and at 60 DAS, respectively) (Table 3). From the results of the field experiment, it could be concluded that intercropping of sorghum, sunflower and sesame with cotton significantly suppressed the density and dry matter production of weeds and produced higher weed control efficiency. The preemergence application of mango leaves extract at 30 per cent spray markedly reduced the growth of weeds when compared to control. Hence, it can be concluded that intercropping along with mango leaf extract spray can be followed for effective and environment friendly weed management in cotton.

\section{Acknowledgement}

With this regard I offer my deep debt of gratitude to my beloved chairperson of the advisory committee, Dr. G. Srinivasan, Professor and Head, Department of Agronomy for his expound guidance, untiring attention, meticulous care, sustained help, constructive criticism, diligent encouragement, painstaking efforts in going through the manuscript and benevolence approach throughout the study to embellish this thesis. I owe a great to him for all the encouragements.

\section{References}

Aladakatti, Y.R., S.S. Hallikeri, R.A. Nandagavi, A.Y. Hugar and N.E. Naveen. 2011. Effect of intercropping of oilseed crops on growth, yield and 
economics of cotton (Gossypium hirsutum) under rainfed conditions. Karnataka J. Agri. Sci. 24(3): 280-282.

El-Rokiek, G. Kowthar, R. Rafat, El-Masry, K. Nadia, Messiha and Salah A. Ahmed. 2010. The Allelopathic effect of mango leaves on the growth and propagative capacity of purple nutsedge (Cyperus rotundus L.). J. Am. Sci., 9: 151-159.

Iqbal, J., and Z.A. Cheema. 2009. Response of purple nutsedge (Cyperus rotundus L.) to crop extracts prepared in various solvents. Allelopathy J., 23(2): 450-452. Iqbal, J., Z.A. Cheema and M. An. 2007. Intercropping of field crops in cotton for management of purple nutsedge (Cyperus rotundus L.). Plant Sci., 300: 163-171.

Nithya, C. and C. Chinnusamy. 2013. Evaluation of weed control efficacy and seed cotton yield in Trangenic cotton Indian J. Applied Res., 3(6): 10-12.

Oliveira, A.M., P.S.L. Silva, C.C. Albuquerque, C.M.S.B. Azevedo, M.J. Cardoso and O.F. Oliveira. 2011. Weed control in corn via intercropping with gliricidia sown by broadcasting. Planta Daninha, 29(3): 535-543.

Rudramuni, M.S., V.K. Rao and V. Ravindra. 2006. Allelopathic Potential of Neem Extract in Horticultural Crops. 8th World Congress of Soil Sci., July 9- 15, Philadelphia, Pennsylvania, USA.

Singh, M. and K.D. Kokate. 2010. Weed management and its effect on cotton (Gossypium hirsutum L.). Annals Plant Prot. Sci., 18(2): 484-487.

Tunio, S.D., M. Ajmal, M.M. Jiskani and G.M. Tunio. 2003 Effect of weed management practices on weeds and cotton yield. Pak. J. Agric. Agril. Eng. Vet. Sci., 19: 29-35.

\section{How to cite this article:}

Sathishkumar, A., G. Srinivasan, T. Ragavan, S. Thiyageshwari and Aananthi, N. 2017. Allelopathic Effect of Different Intercropping System and Tree Leaf Extract Spray on Weed Density, Dry Matter and Weed Control Efficiency in Irrigated Cotton. Int.J.Curr.Microbiol.App.Sci. 6(6): 1322-1329. doi: https://doi.org/10.20546/ijcmas.2017.606.155 